\title{
Numerical simulation of incompressible laminar fluid flow in tubes with wire coil inserts
}

D. Muñoz-Esparza ${ }^{1}$, J. Pérez-García ${ }^{1}$, E. Sanmiguel-Rojas ${ }^{2}$, A. García-Pinar ${ }^{1} \&$ J. P. Solano-Fernández ${ }^{1}$

${ }^{1}$ Department of Thermal and Fluids Engineering, Technical University of Cartagena, Spain

${ }^{2}$ Department of Mechanical and Mining Engineering,

University of Jaén, Spain

\begin{abstract}
Numerical simulations of steady incompressible laminar 3D fluid flow with heat transfer in smooth round tubes with wire coil inserts have been accomplished. Four wire coils with different geometrical characteristics of dimensionless pitch $(p / d)$ and wire-diameter (e/d) have been studied for Reynolds numbers lower than 500-700 using the commercial package software FLUENT.

The numerical results have been compared with experimental data previously obtained by the authors and a good agreement is reached. These results allow one to know the internal flow structure, to identify the critical Reynolds number of oscillating laminar flow and to obtain practical correlations of the Fanning friction factor. So, the internal flow structure under isothermal conditions has been analyzed and a set of practical correlations of Fanning friction factor have been obtained. The heat transfer enhancement produced by the insertion of helical-wire-coils has also been treated. The preliminary results obtained for uniform heat flux are showed. The developed numerical methodology can be used to assess the heat transfer enhancement produced by other kinds of insert devices, to reduce the experimental efforts and to obtain more reliable correlations for heat exchangers design process.
\end{abstract}

Keywords: wire coil inserts, pressure drop, heat transfer enhancement, heat exchangers. 


\section{Introduction}

Heat transfer processes of viscous fluids usually take place in laminar or transitional regimes, where transfer rates are particularly low. Heat exchangers working under these flow conditions are usually candidates to undergo an enhancement technique. Among the different techniques that are effective to improve the thermo-hydraulic behaviour in the tube-side in single-phase laminar flow, the insert devices stand out. The dominant literature (Bergles [1], Webb and Kim [2]) usually mentions five types: wire coils, twisted tapes, extended surface devices, mesh inserts and displaced elements. One of the main advantages of insert devices with respect to other enhancement techniques such as the artificial roughness by mechanical deformation or internal fin types is that they allow an easy installation in an existing smooth-tube heat exchanger.

Due mainly to their low cost, the insert devices which are most frequently used in engineering applications are wire coils and twisted tapes. Wire coil inserts are devices whose reliability and durability are widely contrasted. This is a cheap enhancement technique which is completely viable for many industrial applications, however, the studies in laminar regime are scare in open literature. This fact hinders a widespread use of wire coil inserts in industrial heat exchangers.

It is essential to understand the flow pattern in order to determine how the different flow structures induced by the wire coil augment the pressure drop, because the global improvement is based on the heat transfer/pressure drop ratio. A first experimental approach was carried out by Ravigururajan and Bergles [3] using injection of colored dye. Garcia et al [4] made a more complete study using hydrogen bubbles visualization technique and they stabilised the flow pattern qualitatively.

The necessity to obtain more accurate results and a wider range of information than qualitative visualization techniques has led in an effort to develop a numerical methodology that allow to study the flow pattern thoroughly. Mokamati et al [5] tried to carry out this task in turbulent flow but they were not successful.

In this work, a numerical methodology to study the thermo-hydraulic characteristics in laminar regime has been developed. Numerical results have been compared with experimental data that have allowed to validate the numerical methodology. Friction factor correlations have been obtained and have been compared with experimental data previously obtained by the authors for laminar region [6]. Flow pattern has been analyzed and compared with visualization results, and finally, preliminary results of heat transfer are being obtained.

\section{Numerical simulation methodology}

\subsection{Mathematical model}

The fluid flow studied is governed by 3D incompressible steady-state form of the Navier-Stokes equations. They may be written in Cartesian tensor notation as: 


$$
\begin{gathered}
\frac{\partial}{\partial x_{i}}\left(\rho u_{i}\right)=0 \\
\frac{\partial}{\partial x_{i}}\left(\rho u_{i} u_{j}\right)=-\frac{\partial p}{\partial x_{i}}+\frac{\partial}{\partial x_{j}}\left[\mu\left(\frac{\partial u_{i}}{\partial x_{j}}+\frac{\partial u_{j}}{\partial x_{i}}\right)-\frac{2}{3} \mu \delta_{i j} \frac{\partial u_{k}}{\partial x_{k}}\right]=-\frac{\partial p}{\partial x_{i}}+\frac{\partial \tau_{i j}}{\partial x_{j}} \\
\frac{\partial}{\partial x_{i}}\left(\rho u_{i} E\right)=-\frac{\partial\left(p u_{i}\right)}{\partial x_{i}}+\frac{\partial\left(\tau_{i j} u_{i}\right)}{\partial x_{j}}+\frac{\partial}{\partial x_{i}}\left(k \frac{\partial T}{\partial x_{i}}\right)
\end{gathered}
$$

These equations are solved using FLUENT software, applying the finite volume method to the computational domain described in the following section.

\subsection{Geometry and mesh}

The computational domain is composed by a round smooth tube and a wire coil inserted. The resultant arrangement (a) and a sketch of the wire coil parameters in a longitudinal section (b) are shown in fig. 1.

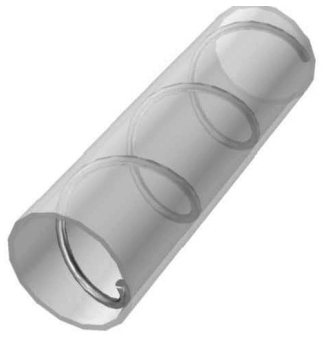

(a)

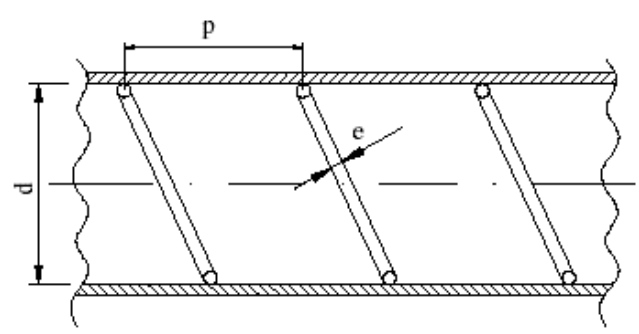

(b)

Figure 1: (a) Studied geometry. (b) Sketch of a wire coil inserted inside a smooth round tube.

The numerical study was carried out on four wire coils fitted in a smooth tube. Table 1 shows geometrical parameters of the wire coils analyzed.

A structured, non-uniform grid has been built through meshing software Gambit. The most important gradients are expected to appear in surroundings of the wire coil surface. For this reason, the region close to the wire has been meshed with a high density of elements and a coarse mesh has been set out for the rest of the section. Simulations have been accomplished to 16 pitches tube lengths meshes for all the wire coils analyzed.

Table 1: Geometrical characteristics of the wire coils studied.

\begin{tabular}{|c|c|c|c|c|}
\hline Wire coil & $d(\mathrm{~mm})$ & $p / d$ & $e / d$ & $p / e$ \\
\hline M01 & 18 & 1.17 & 0.074 & 15.76 \\
\hline M02 & 18 & 2.68 & 0.081 & 33.32 \\
\hline M03 & 18 & 1.86 & 0.099 & 18.75 \\
\hline M04 & 18 & 1.41 & 0.101 & 13.91 \\
\hline
\end{tabular}


In order to ensure accurate results, a grid dependence study has been performed using the Richardson extrapolation method improved with Roache's Grid Convergence Index [7]. This study has allowed to determine the uncertainty of numerical results and the optimum mesh taking into account computational cost and reliability of results simultaneously. The uncertainty propagation has been evaluated according to the procedure detailed in [8]. Expanded uncertainty calculations based on a $95 \%$ confidence level showed limit values of $2.65 \%$ for Reynolds number, $1.44 \%$ for friction factor and $3.32 \%$ for Nusselt number.

\subsection{Simulation hypothesis}

The governing equations have been solved using FLUENT software. The segregated solver and implicit formulation have been chosen to study the flow behaviour under isothermal conditions. SIMPLE, QUICK and first-order upwind schemes have been used in order to discretize pressure-velocity coupling, momentum and energy equations. Non-isothermal flow conditions have been solved using coupled solver and implicit formulation with a second-order flow discretization.

Tube wall inner surface and wire coil were ascribed no-slip and adiabatic boundary conditions for the isothermal flow type. For non-isothermal flow condition, constant heat flux of $q "=11300 \mathrm{~W} / \mathrm{m}^{2}$ over the tube wall inner surface was applied. A free stream flow with uniform temperature and velocity profiles was imposed on the inlet transverse plane and zero diffusion flux was applied to the exit transverse plane for both cases. Propylene-glycol has been used in the numerical simulations, being its physical properties modelled as temperature functions.

\section{Results and discussion}

\subsection{Friction factor results}

Fanning friction factor has been obtained by means of the following expression:

$$
f=\frac{\Delta p d^{5} \pi^{2} \rho}{32 L \dot{m}^{2}}=\frac{\frac{\Delta p}{L} d^{5} \pi^{2} \rho}{32\left(\rho U \frac{\pi d^{2}}{4}\right)^{2}}=\frac{\frac{\Delta p}{L} d^{3} \rho}{2 \mu^{2} R e^{2}}
$$

Where $\Delta p / L$ is the pressure drop per unit length for fully developed flow, $\rho$ is the fluid density and $\mu$ is the fluid dynamic viscosity. These values have been calculated using an area-weighted average in the transverse planes where the pressure drop has been evaluated.

Reynolds numbers from $R e=1$ up to transition Reynolds number to oscillating laminar flow, $R e_{\text {trans }}$, have been analyzed. Friction factor shows a linear tendency in double-logarithm axis. For this reason, it is interesting to obtain correlations for friction factor as a function of Reynolds number using a linear regression $\left(r^{2}=0.99\right.$ for wire coil M03). Reynolds numbers lower than $R e=50$ are very improbable to appear in real situations and are not useful, therefore, numerical 
correlations have been obtained for $R e=50$ to $R e_{\text {trans }}$. In fig. 2 numerical results, numerical correlation and experimental data are compared for wire coil M03. Differences lower than $2.5 \%$ have been obtained comparing numerical and experimental results for the same Reynolds number.

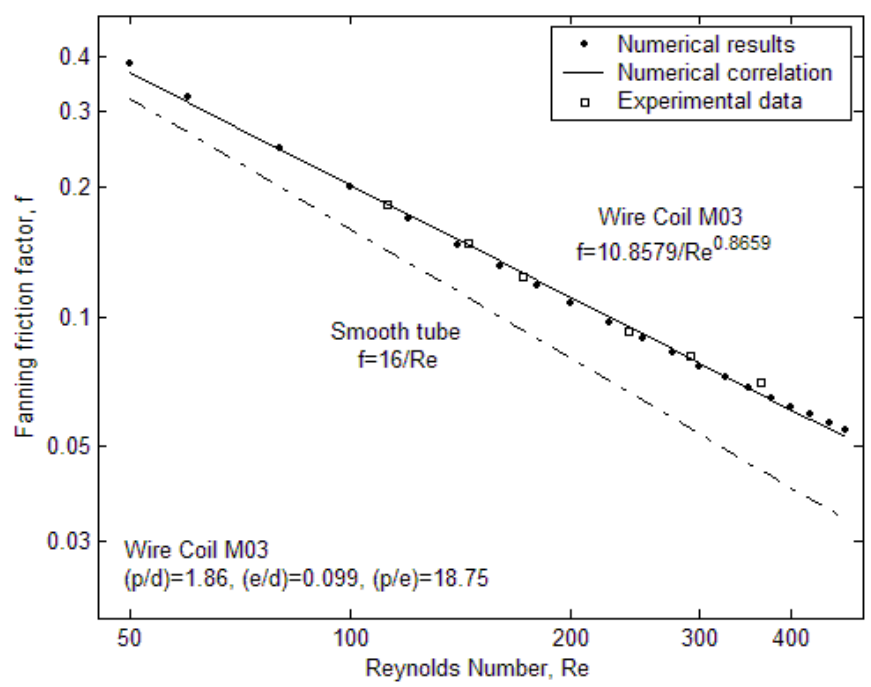

Figure 2: Numerical results, numerical correlation and experimental data for friction factor in wire coil M03.

Numerical correlations verify the form $f=a / R e^{b}$, where $a$ and $b$ are coefficients that depend on the wire geometry. The uncertainty level associated with the friction factor correlations, $f$, and the coefficients, $a$ and $b$, have been computed according to [9]. Expanded uncertainty of $2.9 \%$ has been obtained for wire coil M03 friction factor correlation. Friction factor correlations for the four wire coils are summarized in table 2, with their uncertainties and range of application.

Table 2: $\quad$ Friction factor correlations in laminar regime for the wire coils studied.

\begin{tabular}{|c|c|c|}
\hline Friction factor correlation & Range of application & $\begin{array}{c}\text { Uncertainty of } \\
\text { correlation }\end{array}$ \\
\hline$f_{M 01}=9.2483 / \mathrm{Re}^{0.8284}$ & $50<\mathrm{Re}<500$ & $u_{f, M 01}=3.5 \%$ \\
\hline$f_{M 02}=11.6590 / \mathrm{Re}^{0.9118}$ & $50<\mathrm{Re}<700$ & $u_{f, M 02}=2.6 \%$ \\
\hline$f_{M 03}=10.8579 / \mathrm{Re}^{0.8659}$ & $50<\mathrm{Re}<475$ & $u_{f, M 03}=2.9 \%$ \\
\hline$f_{M 04}=9.5783 / \mathrm{Re}^{0.8167}$ & $50<\mathrm{Re}<475$ & $u_{f, M 04}=3.6 \%$ \\
\hline
\end{tabular}


Heat exchangers do not work under isothermal conditions and it can be possible that this fact modifies the friction factor value. Numerical simulations applying a constant heat flux over the tube wall have been accomplished to assess this effect and friction factor has been calculated in this case, $f^{*}$. For low Reynolds numbers there are no differences between correlations, but when the Reynolds number is increased, the differences grow reaching a maximum value of $2.8 \%$ for $R e_{\text {trans }}$.

These results state that the correlations presented in table 2 are reliable for low Reynolds numbers and can be used for higher Reynolds numbers with a negligible error, which is of the order of its uncertainty.

\subsection{Flow structure}

The internal flow structure can be analyzed evaluating the velocity components in certain planes. The flow in a smooth round tube only has an axial component (theoretical Hagen-Poiseuille velocity profile), but the wire coil inserted modifies the internal flow structure. The wire coil induces a swirl effect on the main axial flow. In fig. 3, azimuthal velocity contours and vectors on a transverse plane are shown. It can be observed that the swirl effect is produced in the wire direction development (negative azimuthal velocities). In the region close to the wire coil surface the flow presents a small zone with an opposite swirl direction. This is due to the fact that main axial flow tries to return the flow that collides with the wire geometry to its previous position. The first swirl effect (negative azimuthal velocities) is more important and it affects to a cylindrical layer that grows towards the tube centreline when the Reynolds number increases. Wire geometries with small values of $(p / d)$ imply a high swirl.

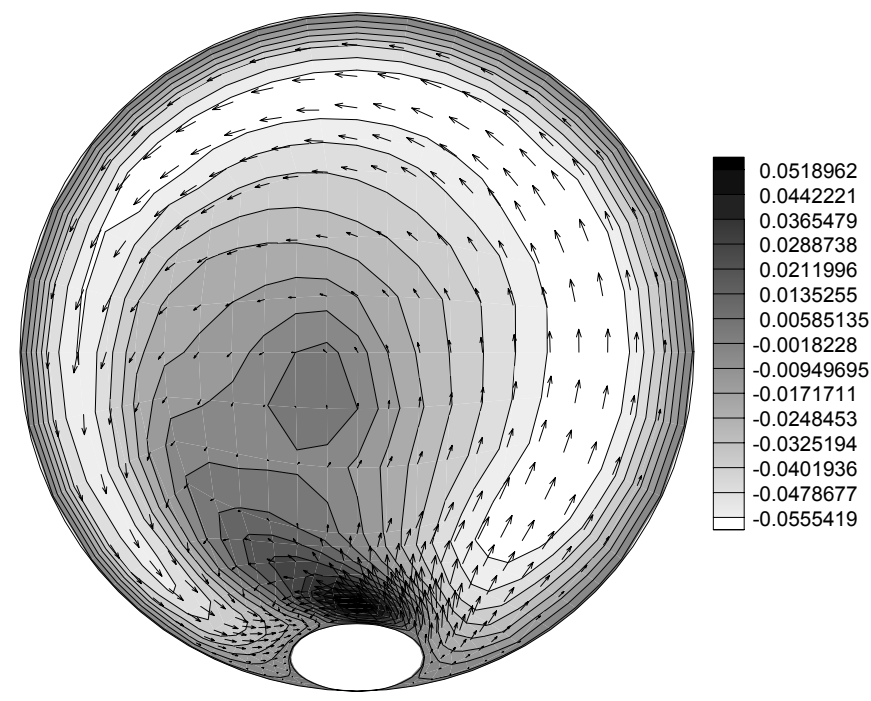

Figure 3: Azimuthal velocity contours $(\mathrm{m} / \mathrm{s})$ and azimuthal velocity vectors on a transverse plane for a $\mathrm{Re}=300$ (flow entering on the sheet plane). 
These results have been compared with experimental data obtained by Ravigururajan and Bergles [3] and García et al [4], concluding that numerical methodology reproduce the swirl effect appropriately and allow to obtain a wider range of information than with experimental techniques.

A recirculation zone just downstream of the wire coil appears for Reynolds numbers slightly higher than $R e=50$ and its characteristic size enlarges when the Reynolds number increases. The recirculation zone size depends principally on the wire-diameter, so, wire coils with high values of $(e / d)$ will present larger recirculation zones. When the transition Reynolds number, $R e_{\text {trans }}$, is exceeded, the recirculation zone which has reached its maximum size, becomes unstable and the flow makes itself oscillating. This unstability is detected by means of numerical divergences that appear during the iterative process, as the algorithm used for the laminar zone is steady and this other region has a non-stationary behaviour. It has allowed to know the $R e_{\text {trans }}$ for the set of wire coils studied, which depends on the wire geometry. For the smooth tube, this transition has been studied experimentally by García et al [4] and it is produced for $R e=1800$ approximately. The wire coil inserted advances the oscillating flow region to lower Reynolds numbers in the order of $R e=500-700$ for the wire coil geometries analyzed on this work. In fig. 4 axial velocity contours and vectors are shown for two different Reynolds numbers on a longitudinal plane. In fig. 4 (a) it can be observed that there are no recirculation zone for low Reynolds numbers $(\operatorname{Re}<50)$ and fig. $4(\mathrm{~b})$, where the recirculation zone takes place $(R e=300)$. This structure agrees with experimental results showed in [4].

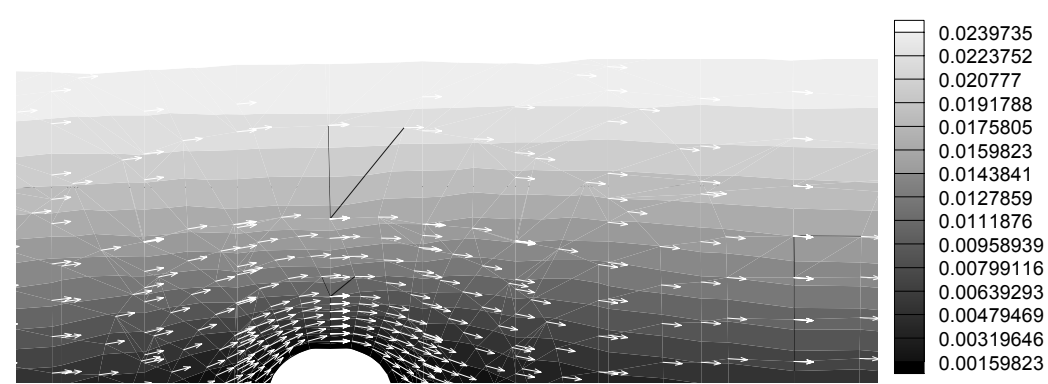

(a) $\mathrm{Re}=10$

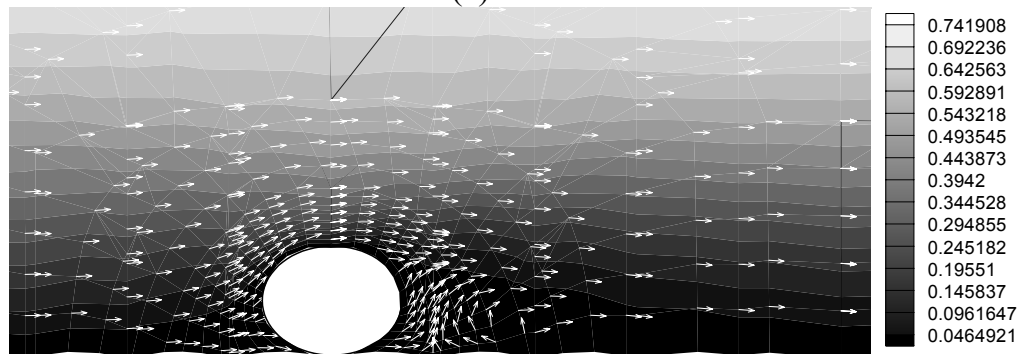

(b) $\mathrm{Re}=300$

Figure 4: Axial velocity contours $(\mathrm{m} / \mathrm{s})$ and axial velocity vectors on a longitudinal plane. Detail round the wire coil geometry. 


\subsection{Heat transfer results}

Preliminary results of heat transfer have been obtained. Nusselt number has been calculated from eqn. (5).

$$
N u=\frac{d}{k} \frac{q^{\prime \prime}}{T_{\text {wall }}-T_{\text {flow }}}
$$

Where $k, T_{\text {flow }}$ and $T_{\text {wall }}$ are the mean values of thermal conductivity, flow temperature and inner wall temperature in a transverse plane, respectively. In fig. 5 numerical results of Nusselt number, experimental data and Churchill and Ozoe smooth tube correlation [10] are compared. Nusselt number is increased in the order of 1.7 times in respect with the smooth tube and this augment is almost independent of the Reynolds number for the laminar forced convection zone, showing these values a similar trend to the smooth tube. There are slight differences between numerical and experimental results, which are due to the different methodologies used to evaluate the Nusselt number.

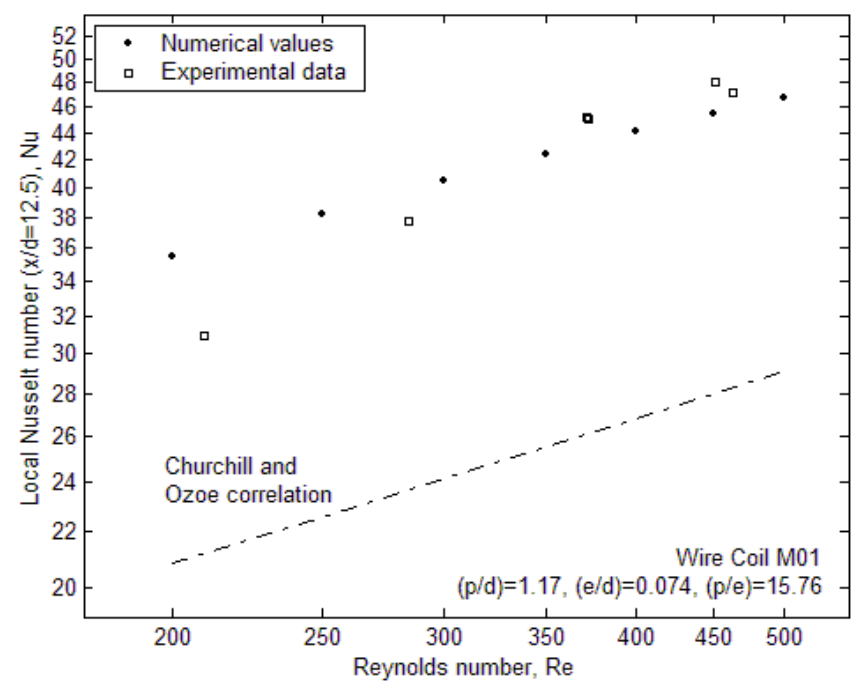

Figure 5: $\quad$ Numerical results and experimental data for the Nusselt number in wire coil M01 for a longitudinal dimensionless distance of $x / d=12.5$.

\section{Conclusions}

1. A numerical methodology to study the laminar fluid flow in tubes with wire coil inserts has been developed. The numerical results have been compared with experimental data obtained by the authors and a good agreement has been reached. Differences lower than $2.5 \%$ for the friction factor have been obtained comparing numerical and experimental results, which has allowed one to validate the numerical methodology. 
2. The friction factor increases in reference to the smooth tube due to a coupled effect between the pitch $(p / d)$ and the wire-diameter $(e / d)$. Small pitches and big wire-diameter geometries provokes an augmentation of friction factor for constant Reynolds numbers.

3. A set of practical and reliable correlations for the friction factor has been obtained as a function of the Reynolds number and their associated uncertainty has been calculated.

4. Simulations under isothermal and non-isothermal conditions have been made to study the effect of an applied heat flux over friction factor results. Slight differences appear between correlations, which are similar to the correlations uncertainties. By this reason, correlations showed in table 2 can be used in order to predict the friction factor with a negligible error.

5. The internal flow structure has been assessed. It is composed by a main axial flow with a swirl effect induced by the wire coil and a recirculation zone just downstream of the wire. The pitch determines the swirl effect, higher when it is reduced, and the wire-diameter determines the size of the recirculation zone, bigger when it is increased. The size of the recirculation zone enlarges and azimuthal velocity component rises when the Reynolds number augments. The recirculation zone becomes unstable for Reynolds numbers between 475-700 ( $\left.R e_{\text {trans }}\right)$, depending on the wire coil geometry. These instabilities advance the transition region to lower Reynolds numbers in reference to the smooth tube.

6. Heat transfer has also been treated. Preliminary results show that wire coils generate a heat transfer enhancement but it is necessary to evaluate the global improvement taking into account the pressure drop. Different geometries are being studied and a set of correlations for Nusselt number will be obtained (in progress).

\section{Acknowledgements}

This research has been partially financed by the DPI 2007-66551-C02-01 grant of the "Dirección General de Investigación del Ministerio de Educación y Ciencia de España'.

\section{References}

[1] Bergles, A.E., Techniques to augment the heat transfer, Handbook of Heat Transfer Applications, McGraw Hill, New York, 1985.

[2] Webb, R.L. \& Kim, N.H., Principles of Enhanced Heat Transfer, second ed., Taylor \& Francis Group, New York, 2005.

[3] Ravigururajan, T.S. \& Bergles, A.E., Visualization of Flow Phenomena Near Enhanced Surfaces. Journal of Heat Transfer (Transactions of the ASME), 116, pp. 54-57, 1994.

[4] García, A., Solano, J.P., Vicente, P.G. \& Viedma, A., Flow pattern assessment in tubes with wire coil inserts in laminar and transition regimes. International Journal of Heat and Fluid Flow, 28, pp. 516-525, 2007. 
[5] Mokamati, S.V. \& Prasad, R.C., Numerical Simulation of Fluid Flow and Heat Transfer in a Concentric Tube Heat Exchanger. Proc. of the $19^{\text {th }}$ Canadian Congress of Applied Mechanics: Calgary, 2003.

[6] García, A., Solano, J.P., Vicente, P.G. \& Viedma, A., Enhanced of laminar and transitional flow heat transfer in tubes by means of wire coil inserts. International Journal of Heat and Mass Transfer, 50, pp. 3176-3189, 2007.

[7] Freitas, C.J., Ghia, U., Celik, I., Roache, P. \& Raad, P., ASME's quest to quantify numerical uncertainty. $41^{\text {st }}$ AIAA Aerospace Sciences Meeting and Exhibit: Reno, 2003.

[8] ISO, Guide to the Expression of Uncertainty in Measurement, first ed., International Organization for Standarization, Switzerland, 1995.

[9] Coleman, H.W. \& Steele, W.G., Experimentation and Uncertainty Analysis for Engineers, second ed., John Wiley \& Sons Inc., New York, 1999.

[10] Churchill, S.W. \& Ozoe, H., Correlations for forced convection with uniform heating in flow over a plate and in developing and fully developed flow in a tube. Journal of Heat Transfer, 95, pp. 78-84, 1973. 